\title{
Situando las historias de la arqueología (desde Latinoamérica)
}

Carlo E. Piazzini Suárez*

Recibido:

14 de marzo de 2019

Aceptado:

4 de julio de 2019

\section{Resumen}

Este artículo ofrece una revisión de literatura producida en las últimas décadas sobre historia de la arqueología en Europa, Norteamérica y Latinoamérica. Mediante un ejercicio situado desde ésta última región e interesado por las geografías del conocimiento, se propone un análisis crítico de las historias de la arqueología, que permite identificar regímenes históricos y espaciales que subyacen a una parte importante de las narraciones del devenir de la disciplina. Así mismo, se indaga por el lugar que en estas narraciones ocupan las historias de la arqueología en Latinoamérica y cómo desde la región se valoran sus relaciones con las trayectorias de la arqueología en otras partes. Se propone, finalmente, una geografía de las historias de la arqueología que, superando la imaginación geográfica moderna, sea sensible a espacialidades más fluidas, abiertas y discontinuas, que han incidido en la conformación de aquello que hoy llamamos arqueología.

\section{Placing the histories of archaeology (from Latin America)}

\begin{abstract}
This paper presents a review of relevant literature about the history of archaeology in Europe, North America and Latin America produced in the last decades. Through an exercise situated in this last region, and with an interest in the geographies of knowledge, we propose a critical analysis of the histories of archaeology, to identify the historical and spatial regimes that underlay most of the narratives about the becoming of the discipline. Also, we evaluate the role of those narratives in the histories of Latin American archaeology and how its relationships with the trajectories of archaeology in other regions are assessed from Latin America. Finally, we propose a geography of the histories of archaeology, which overcomes the imagination of modern geography, to be sensitive to more fluid, open, and discontinuous spatialities, that have influenced the configuration of that we call archaeology today.
\end{abstract}

Palabras clave

Historiografía Latinoamérica Geografía de las ciencias Historia de las ciencias
Keywords

Historiography Latin America Geography of sciences History of sciences 


\section{Introducción}

Recientemente Haber y Roberts (2014, p. 3411) han sugerido que la arqueología es una disciplina obsesionada con su historia: "De hecho, - dicen - los libros de texto, manuales, síntesis regionales, libros sobre teoría, y otros, darán en las primeras páginas la bienvenida con una historia arqueológica sobre un tema determinado". Esta apreciación da la idea de que historizar la praxis disciplinar ha ocupado un lugar significativo en el ejercicio de la arqueología. Es claro, tal y como señalaba Kuhn hace más de cuatro décadas, que poner un "poco de historia" en las introducciones de los libros de texto, es un recurso frecuente para hacer sentir a los discípulos y profesionales partícipes de una tradición científica (Kuhn, 1970, p. 137). Pero aparte de este recurso pedagógico y narrativo, hasta la década de 1980 las publicaciones expresamente orientadas a la historia de la arqueología eran relativamente pocas (Moro, 2012, p. 180). Por ejemplo, un análisis sobre literatura arqueológica producida en Norteamérica entre 1948 y 1985 indicaba 7.000 registros, de los cuales sólo 300 correspondían a referencias de interés "metaarqueológico", uno de cuyos temas era la historia de la arqueología (Embree, 1993, p. 290).

Desde la década de 1980, las publicaciones que explícitamente se orientan hacia los estudios históricos de la arqueología han aumentado notablemente, de tal modo que Trigger (1994), indicaba ya la dificultad de dar cuenta de la bibliografía existente. En Europa y el mundo anglosajón, la historia de la arqueología se identifica hoy como un campo específico de investigación y debate, con una revista dedicada a ello (Bulletin of the History of Archaeology), varios textos enciclopédicos (Bahn, 1996, 2014; Murray, 1999, 2001, 2007a), y un programa de sistematización y preservación de archivos de alcance continental (Archives of European Archaeology - AREA-) (Murray y Evans, 2008; Schlanger y Nordblach, 2008a). En Latinoamérica, desde por lo menos la primera mitad del siglo XX se han venido escribiendo algunas historias nacionales y, en las últimas tres décadas, narraciones más o menos detalladas acerca de lo que ha sido el devenir de la disciplina, han acompañado con frecuencia los ejercicios reflexivos sobre su presente y futuro (Politis, 2003; Schávelzon, 2006).

Dado este auge relativo, se ha dicho, en tono celebratorio, que la historiografía de la arqueología ha alcanzado su "mayoría de edad" al ser un tema que, por derecho propio, hace parte de la agenda disciplinar (Kaeser, 2008, p. 9; Moro, 2012, p. 178; Murray, 2007b, p. xvii; Murray y Evans, 2008, p. 3; Trigger, 1994, 2001, p. 639). Esta idea genera la percepción de que el ejercicio sustantivo de la disciplina antecede epistémicamente al ejercicio de su historización y que ambos transcurren de manera acumulativa y perfectible. Sin embargo, estas historias no tratan con un objeto de estudio dado, sino que han contribuido, ellas mismas, a la construcción de lo que se denomina arqueología (Díaz, 2007, p. 3). Más allá de celebrar su mayoría de edad, hay que advertir que resultan fundamentales para legitimar y dar continuidad, o para criticar y transformar, determinadas arqueologías. Igualmente, sirven para marcar límites entre lo que se considera científico o no y para consagrar, excluir, minimizar o reivindicar a ciertos actores en su participación en el desarrollo de particulares tradiciones científicas y académicas (Lepenies y Weingart, 1983).

De ahí la relevancia de análisis historiográficos propiamente dichos (Tucker, 2008), que más allá de referenciar el acumulado de publicaciones sobre historias de la arqueología, permitan identificar cómo han sido elaboradas esas historias, quienes las han hecho, desde dónde, con qué propósito y cuáles han sido sus resultados. Así entendidos, los estudios historiográficos de la arqueología son relativamente pocos y han sido elaborados, sobre todo, desde aquellos lugares en los que se suelen situar los orígenes y centros más importantes de desarrollo de la disciplina (Christenson, 1989; Corbey y Roebroeks, 2001; Díaz y Sørensen, 1998; Haber y Roberts, 2014; Kaeser, 2002, 2008; 
Meltzer, 1989; Moro, 2006, 2010, 2012, 2013; Murray, 2002; Murray y Evans, 2008; Ruíz, 2017; Schávelzon, 2006; Schlanger, 2002, 2004; Schlanger y Nordblach, 2008b; Trigger, 1994, 2001; Trigger y Glover, 1981).

El presente artículo propone un mapeo de las historias de la arqueología de Latinoamérica, a partir de una revisión de la literatura disponible para las últimas décadas, llamando la atención acerca de una geografía de las ciencias (Livingstone, 2003), y más ampliamente del conocimiento (Agnew, 2007). Así como en las historias de la arqueología operan particulares concepciones del tiempo que corresponden a unos "regímenes historiográficos" (Hartog, 2007, p. 37), también funcionan unos "regímenes espaciales" que aquí asimilamos a imaginarios geográficos (Gregory, 2009), los cuales implícita o explícitamente ordenan las trayectorias históricas en particulares relaciones espaciales. Esta incorporación de una especialización crítica (Soja, 1989, p. 12) a la historia y los estudios de la ciencia, es relativamente reciente (Livingstone, 2003; Ophir y Shapin, 1991), y supone por lo tanto una agenda de trabajo que requiere de estudios particulares y, para el caso, de unas geografías de la arqueología ${ }^{1}$.

El artículo se compone de cuatro partes. Primero, se espacializan las historias generales de la arqueología, identificando el imaginario geográfico que subyace a sus estructuras narrativas y justifica sus lugares de enunciación. Segundo, se analiza el lugar que en éstas historias ocupa la arqueología hecha en Latinoamérica. Tercero, se analizan diferentes formas de concebir las historias de la arqueología en Latinoamérica y su relación con las arqueologías que se han hecho en otras partes. Finalmente, se ofrece una síntesis de los principales regímenes históricos y espaciales que soportan las visiones sobre el devenir de la disciplina y se indican los beneficios que la adopción de enunciados provenientes de la geografía de las ciencias, tendrían para ejercicios menos introspectivos de historia de la arqueología.

\section{Historia e historias de la arqueología}

La idea de "paisajes teóricos", para indicar la coexistencia de diferentes trayectorias de la arqueología (Hegmon, 2003, p. 214; Politis, 2003; Preucel y Hodder, 1996, p. 6), supondría que, en el tránsito desde una Historia a unas historias de la arqueología, se reconoce expresamente una dimensión geográfica del conocimiento. Pero se trata de una metáfora, que aprovecha el sentido de simultaneidad que evocan los paisajes para tratar de ordenar una multiplicidad de formas de hacer arqueología, que ya no es fácil integrar en una Historia única.

Hasta hace cuatro décadas, las narraciones sobre historia de la arqueología fijaban sus teatros principales en Europa occidental y Norteamérica, siendo las trayectorias en otras regiones del planeta, replicas o reflejos más o menos tardíos, más o menos imperfectos de las primeras. Como lo advirtió Trigger a propósito de la arqueología procesual en Norteamérica: "La arqueología americana fue vista como siguiendo un patrón lógico y en última instancia inevitable, el cual, en ausencia de interferencias políticas o anomalías sociales, podría trazar el desarrollo de la arqueología en cualquier parte" (Trigger, 2001, p. 633). Así es que obras, como Idea of Prehistory (Daniel, 1963) y A History of American Archaeology (Willey y Sabloff, 1974), se constituyeron en poderosos referentes para comprender la historia de la arqueología en otras geografías. Y por lo menos hasta La historia del pensamiento arqueológico de Trigger (1992), se consideraba que la Historia de la disciplina era una sola. Aunque pudieran reconocerse enfoques "paralelos" o "tradiciones regionales", ello no llegaba a comprometer la organización lineal de una Historia orientada desde lo precientífico a lo científico, y de lo central a lo periférico. Las diferencias espaciales eran un obstáculo a superar en la expansión de la ciencia arqueológica y, por lo tanto, en el desarrollo de su historia.
1. Las geografías del conocimiento remiten ampliamente a las relaciones entre espacio, conocimiento y poder. En este sentido, las geografías de la arqueología se refirieren a aquellas relaciones que operan entre particulares procesos de producción, circulación y reproducción/apropiación de conocimiento arqueológico, y específicas formaciones espaciales como cuerpos, localizaciones, territorios, arquitecturas y redes. Estas relaciones son dialécticas: los conocimientos arqueológicos cuentan entre sus condiciones de posibilidad con esas formaciones espaciales pero, a su vez, pueden contribuir a darles forma o a fundamentarlas. Para el estudio de las geografías de la arqueología, resulta útil aproximarse a las dinámicas de localización, distribución y movilización geográfica de los conocimientos, así como su diferenciación y jerarquización conforme a esquemas geopolíticos (Piazzini Suárez, 2010). 
La fuerza de este régimen espacial no es fácil de superar. Trigger, pionero de lo que llamó una "historia social de la arqueología", tuvo dificultades para definir la estructura de su "historia del pensamiento arqueológico":

(...) el presente estudio - decía- no tratará las diversas tendencias de interpretación arqueológica desde una perspectiva específicamente cronológica, geográfica o subdisciplinaria... al contrario, intentará investigar una serie de orientaciones interpretativas en el orden más o menos cronológico en el que se originaron (Trigger, 1992, p. 23; cursivas agregadas).

Este "más o menos cronológico" correspondía a la incomodidad de mantener una perspectiva lineal para narrar una historia que empezaba a reconocerse plural, pero a la vez, al riesgo de fragmentarla como consecuencia de múltiples trayectorias regionales y locales.

Otros esfuerzos pioneros por tratar de dar adecuado tratamiento a esta pluralidad se encuentran en la compilación efectuada por Daniel (1981) y los proyectos editoriales de la revista World Archaeology (1981, 1982), bajo la edición de Trigger y Glover (1981). En la primera se incluyeron, en clave de "historia social", biografías y tradiciones de países tan diversos como Dinamarca, Francia, Checoslovaquia, Canadá, Nueva Zelanda, India y regiones como Europa e Iberoamérica. En la segunda se hicieron visibles tanto tradiciones regionales (angloamericana, escandinava, del Cercano Oriente y latinoamericana), como nacionales (Francia, Holanda, Israel, Unión Soviética, China, Japón, India, Australia, Nueva Guinea, México y Perú), sin que la presentación de los trabajos estuviera supeditada a un esquema cronológico. Cabe decir que, de acuerdo con los editores, en las "tradiciones regionales de investigación arqueológica" se observan

(...) variaciones en la naturaleza del registro arqueológico y en los recursos disponibles para la investigación. Pero sobre todo, parecen reflejar diferentes lealtades nacionales o étnicas, adscripciones a filosofías políticas alternativas o tradiciones culturales $y$, algunas veces, la influencia crucial en regiones específicas de eminentes académicos (Trigger y Glover, 1981, p. 133).

Con ello se planteaba tempranamente la existencia de algo semejante a una de geografía histórica de la arqueología, lo cual encontraría eco en los trabajos compilatorios de Ucko (1995) sobre tradiciones regionales (Europa, África y Suramérica) y nacionales (Zimbabue, Alemania, Indonesia, Namibia, India, Australia, Canadá, Brasil, Portugal, Irlanda, Japón, Rusia y Francia), y de Lozny (2011a) sobre Europa, Suramérica y el Caribe, Asia y el Pacífico, y África.

Pero es posible identificar una serie importante de trabajos en los que sigue primando un ordenamiento cronológico, incluso cuando apelan al reconocimiento de la pluralidad de las historias de la disciplina. Díaz (2007) ha enfatizado en el carácter heterogéneo de la arqueología y, en consecuencia, ha criticado el modo no incluyente de la mayoría de las historias de la disciplina, en la medida en que se han centrado en la prehistoria de las grandes civilizaciones o en particulares tradiciones nacionales o imperiales, sobre todo europeas. Pero su iniciativa de "ofrecer una historia comprensiva de la arqueología global, esto es, una que considere todos sus campos a través del mundo, durante el siglo XIX" (Díaz, 2007, p. 23), se organiza de manera explícitamente teleológica: "esta historia - dice- es un recuento histórico de una disciplina que emerge en el siglo XIX y maduró durante el siguiente, o que se desarrolló profesionalmente entre las dos guerras, especialmente luego de la segunda guerra mundial" (Díaz, 2007, p. 3). Por su parte Trigger (2001), precisamente en un balance sobre la historiografía arqueológica, también ha empleado un esquema cronológico, según el cual, con excepción de la las "historias populares" de la arqueología presentes durante todo el siglo XX, existirían 
tres periodos sucesivos: historias tempranas, intelectuales (internalistas) y sociales (externalistas).

A esta dificultad de romper con un esquema fundamentalmente cronológico y teleológico, con frecuencia se asocia la persistencia de una cartografía euro/anglo centrada de la historia de la arqueología. Por ejemplo, Murray (2007b), ha efectuado abiertamente una cancelación del relativismo extremo al que podría conducir una estructura geográfica fuerte de las historias de la arqueología, al optar por una narrativa que va del origen europeo y norteamericano de la arqueología (antes del siglo XIX), pasando por su consolidación en relación con el origen de las naciones y los imperios (siglo XIX), para llegar a una arqueología mundial (siglos XX y XXI). Y en el Oxford Handbook of Archaeology (Cunliffe, Gosden y Joyce, 2009), cualquier mención sobre trayectorias regionales por fuera del mundo anglosajón es accesoria frente a los capítulos iniciales que, centrados en las experiencias británica y norteamericana, y ordenados desde una etapa formativa a una teórica, fungen nuevamente como marcos de referencia para la historia de la arqueología en el resto del mundo (Boast, 2009; Johnson, 2009).

En el tránsito de las compilaciones efectuadas recientemente por Bahn, podría registrarse, a primera vista, una crisis de este régimen histórico y espacial. Mientras la primera se organizaba según un esquema cronológico compuesto por siete periodos o fases (Bahn, 1996), la segunda opta por un esquema regional que incluye tradiciones de la Europa antigua, el mundo egeo, el mundo clásico, Egipto, Asia occidental y del sur, África, el Lejano Oriente, Rusia, Norteamérica, Mesoamérica, Suramérica y Australasia (Bahn, 2014). Pero, tras la aparente importancia de la diversidad regional, esta última obra está estructurada en términos del origen, desarrollo y expansión geográfica de la arqueología desde Europa hacia las demás regiones que se incluyen en el libro. Esta perspectiva también está presente en otra obra de carácter enciclopédico publicada por Murray, en donde los mapas de distribución de los sitios arqueológicos reseñados (Murray, 2007a, pp. xxxiv-iLiii) resultan elocuentes acerca de una memoria arqueológica que, al pretenderse global, está claramente eurocentrada.

Es así que destacadas compilaciones de alcance global publicadas en las últimas dos décadas, más o menos alimentadas por el reconocimiento de la pluralidad de las prácticas que conforman la arqueología en el planeta, y en las cuales incluso se pueden leer voces críticas del colonialismo intelectual, no dejan de ser en su mayoría empresas editoriales administradas desde centros anglosajones, que quisieran con-centrar en, y desde un solo espacio, todos los saberes sobre la arqueología (una renovación del sueño enciclopédico ilustrado). Por lo tanto, no conllevan a una transformación de la Historia de la arqueología, generalmente entendida como un proceso que se inicia en Europa y Norteamérica y luego, con particulares matices, se "difunde" por medio de "influencias" para formar "tradiciones" locales o regionales por el resto del planeta.

Recientemente, Ruiz (2017) ha ofrecido una síntesis de las "tradiciones contemporáneas de historias de la arqueología", compuesta por diferente "corrientes". La tradición anglosajona (Reino Unido, Estados Unidos, Canadá y Australia) se establece como el mainstream indiscutible, mientras que la tradición francesa es un mainstream de segundo nivel. Por otra parte, un mainstream emergente corresponde a la tradición historiográfica de la India, y unas minorities están referidas a las tradiciones española, italiana, escandinava y centroeuropea. El autor, quien habla desde una de éstas últimas, se pregunta si en recientes compilaciones como la de Bahn (2014), que a su juicio representa la "conquista - aunque sea parcial e incompleta - de la memoria de la arqueología en todo el mundo", no hay algo de "colonialismo intelectual". Lo cual resulta irónico, dado que Ruiz evita cualquier referencia a la historiografía de la arqueología en Latinoamérica. 


\section{El lugar de Latinoamérica}

Cualquier referencia a los estudios históricos efectuados por latinoamericanos fue escasa en la literatura internacional hasta la década de 1980, salvo algunas anotaciones sobre el ejercicio de la arqueología en México y Perú, en virtud del carácter monumental de sus materialidades prehispánicas. De ello resulta revelador que en History of American Archaeology (Willey y Sabloff, 1974), la referencia a autores de la región correspondía tan sólo al 11,5\%, de un total aproximado de 500 fuentes bibliográficas (Schávelzon, 2006).

Sin embargo, las narraciones del devenir de la arqueología, hechas desde países latinoamericanos, poseen, una larga trayectoria, que Schávelzon (2006) ha ordenado en etapas. La primera (del siglo XIX a 1960), se habría caracterizado por la elaboración de biografías y necrologías de exploradores y arqueólogos notables, la reedición y/o publicación de documentos antiguos de valor para la arqueología, las crónicas sobre el descubrimiento e investigación de sitios monumentales y la trayectoria de instituciones. En la segunda etapa (1960-1970), se habrían sumado ejercicios retrospectivos, a veces críticos, escritos fundamentalmente por arqueólogos interesados en usar la historia de la disciplina para convalidar nuevos paradigmas. En la tercera etapa (1970-1974), las historias organizadas por países se incrementaron, abriendo el camino para un campo de investigación dedicado a la historiografía arqueológica. Finalmente, aun cuando no define expresamente una cuarta etapa, considera que a partir de la década de 1980 la historiografía ha comenzado a explorar cada vez más las relaciones entre arqueología y poder, en varios frentes: revisiones críticas de las arqueologías nacionales y sus conexiones con sistemas democráticos o regímenes dictatoriales; análisis de la hegemonía de las elites intelectuales a la sombra de las instituciones oficiales; y visibilización del papel jugado por los sectores excluidos, las mujeres y los grupos étnicos.

Por lo menos hasta la década de 1980, estos ejercicios se habían anclado fundamentalmente en espacios nacionales como México (Bernal, 1979), Colombia (Duque, 1955), Argentina (Fernández, 1979) y Perú (Fung, 1965). A partir de entonces, paulatinamente, van incorporándose textos sobre otras historias nacionales o regionales de la arqueología latinoamericana en proyectos editoriales de alcance mundial. Textos dedicados a Iberoamérica y México a cargo de Lorenzo (1981a y 1981b) fueron incluidos en las compilaciones de Daniel (1981) y Trigger y Glover (1981), respectivamente. También en ésta última, se incluyó un artículo sobre Perú a cargo de Schaedel y Shimada (1982), y Schávelzon (1989) participó con un trabajo sobre Mesoamérica en la compilación de Christenson (1989). En la década siguiente Politis (1995) publicó una síntesis regional sobre Suramérica hispana en la compilación de Ucko (1995), en donde también participó Funari (1995) con un trabajo sobre Brasil. Posteriormente, Politis y Pérez (2004) presentaron un texto sobre Latinoamérica en $A$ Companion to Social Archaeology (Meskell y Preucel, 2004). La visibilidad que hasta entonces habían adquirido los textos sobre la historia de la arqueología latinoamericana en Europa puede constatarse en World History of Nineteenth-century Archaeology (Díaz, 2007), específicamente en el apartado sobre arqueología colonial. Sin embargo, destaca la ausencia de mujeres latinoamericanas, tanto en las historias globales con enfoque de género (Díaz y Sørensen, 1998), como en aquellas elaboradas por autores latinoamericanos sobre sus países.

Otros aportes recientes sobre Latinoamérica en obras compilatorias con enfoque histórico y alcance global son los de Funari (2008) sobre Brasil; Martínez, Taboada y Auat (2008) sobre Argentina; Dillehay (2008) sobre Latinoamérica; Funari, Zarankin y Stovel (2009) sobre Suramérica; Cyphers (2014) sobre Mesoamérica; y López (2014) sobre Suramérica. También en varias de las compilaciones enciclopédicas efectuadas 
en los últimos años (Cunliffe, Gosden y Joyce, 2009; Murray, 2001; Smith, 2014), se incluyen entradas para prácticamente todos los países latinoamericanos, con algunos trabajos de corte histórico a escala regional o nacional, e incluso biografías de autores latinoamericanos. Y, por primera vez, en Comparative archaeologies (Lozny, 2011a), se incluyó en una compilación global un apartado robusto para Suramérica y El Caribe, compuesto por seis textos de autores regionales.

Así pues, se ha ido dibujando un lugar para Latinoamérica y algunos de sus países en las narrativas mundiales de la historiografía de la arqueología, administradas sobre todo desde el mundo anglosajón y europeo. De manera simultánea, se generaban algunos proyectos coordinados desde la región o por autores latinoamericanos entre los que destacan: History of Latin American Archaeology (Oyuela, 1994a), quizá el primer trabajo en lengua inglesa enteramente dedicado a la historia de la arqueología en la región; Archaeology in Latin America (Politis y Alberti, 1999), en donde se incluyeron algunos artículos de corte historiográfico; la síntesis regional efectuada por Politis (2003); y la interesante apuesta de Funari, Zarankin y Stovel (2005) en su Global Archaeological Theory, uno de los pocos textos de alcance global convocados desde espacios que no han sido centrales en la geografía de la historiografía arqueológica.

Varios de estos trabajos se inscriben en una dinámica de diálogo que en las últimas dos décadas ha puesto en contacto a quienes hacen arqueología en Latinoamérica, mediante congresos, reuniones y proyectos editoriales (Haber, 2014, p. 6.823). De ello hace parte una serie importante de publicaciones que incluyen de manera más o menos detenida, aproximaciones a las historias nacionales o regionales de la arqueología (p. ej. Curtoni y Politis, 2006; D'Agostino, 1999; Funari, Zarankin y Stovel, 2005, 2009; Gnecco y Ayala, 2010; Herrera, 2013; Jaramillo, 2008; Nastri y Menezes, 2010; Politis y Alberti, 1999; Salerno, 2012; Shepherd, Gnecco y Haber, 2016; Silverman e Isbell, 2008; Zarankin y Acuto, 1999, 2008).

\section{¿Historia latinoamericana de la arqueología?}

Pero, ¿por qué habría de identificarse una particular forma de las historias de la arqueología en este espacio subcontinental que denominados Latinoamérica, Suramérica o Iberoamérica? Su visibilidad depende, en buena medida, del lugar desde el cual se mire. Para quienes hablamos desde la región, es posible que la arqueología latinoamericana resulte autoevidente, no siendo así para quienes hablan desde "afuera", e incluso desde una localización "fronteriza". Dillehay, a medio camino entre Norteamérica y Suramérica, indicaba hace poco: "no podemos decir que haya una verdadera "arqueología latinoamericana (incluso americana)" dada la gran extensión del área y las múltiples tradiciones locales y desigualdades en el acceso a subvenciones, entrenamiento académico y experticia técnica" (Dillehay, 2008, p. 165). No obstante, alcanzaba a identificar rasgos que, aun cuando no exclusivos, consideraba comunes o frecuentes en la región: prevalencia de investigaciones empíricas e inductivas en detrimento de la aplicación de modelos interpretativos y elaboraciones teóricas; ausencia de paradigmas teórico-metodológicos hegemónicos; poca meritocracia en la selección de profesionales; falta de apoyo económico a la investigación; estrecha relación entre arqueología y espacios nacionales; y una situación periférica o marginal frente a la arqueología europea y norteamericana.

Para varios autores latinoamericanos, la existencia de una arqueología regional aparece de manera más nítida, pero ello no quiere decir que estén de acuerdo acerca de los aspectos que la definen, como tampoco en el sentido que a futuro debería adquirir. Desde México, Lorenzo (1981a, p. 60) se refería a una diferencia histórica que habría definido las características de la arqueología entre "los países de habla inglesa al norte 
del río Grande y los países no angloparlantes al sur del mismo". Con un enfoque interesado por la historia de las ideas y los cambios en los patrones de pensamiento, consideró que tanto las evidencias arqueológicas y el acervo documental, como los procesos históricos regionales, habrían definido unos rasgos específicos para las arqueologías iberoamericanas. Ello se hacía visible con particular intensidad en México en el siglo XX, cuando arqueologías vinculadas con el nacionalismo y el indigenismo, más cercanas a la historia que a la antropología, entraban en tensión o conformaban productos eclécticos con las arqueologías norteamericanas. También afirmaba que, desde el siglo XIX, una suerte de "colonialismo arqueológico" había provocado que en países como México y Perú, una oleada de empresarios, diplomáticos, viajeros y científicos extranjeros obtuvieran datos para ganar prestigio en sus países de origen, dejando a cambio, cuando así sucedió, conocimientos que nada tenían que ver con los intereses y percepciones locales sobre lo prehispánico.

Lorenzo formaba parte de un grupo de arqueólogos que desde la década de 1970, en México, Costa Rica, Perú, República Dominicana, Puerto Rico y Venezuela, adoptaron conceptos marxistas y propusieron una "arqueología social". Esta se presentaba como alternativa al auge neopositivista de las arqueologías norteamericanas, buscando "insertar la disciplina en el campo de las ciencias sociales, para alcanzar la reformulación de las bases epistemológicas de la educación y la enseñanza de la historia, lo que consideramos parte fundamental de la conciencia nacional de una sociedad formada e informada por su historia y de su destino como comunidad soberana en el contexto integrado de una nueva Latinoamérica en marcha” (Vargas y Sanoja, 1999, p. 60).

En una perspectiva afín, desde Lima, Lumbreras (1992) sostenía que las teorías y metodologías de la arqueología europea y anglosajona no podían ser transferidas automáticamente para tratar de explicar los procesos históricos suramericanos. En su lugar, era necesario realizar ajustes y desarrollar enfoques propios a partir de la investigación de dichos procesos, pero además, consideraba que los mismos habían imprimido una huella particular en las formas de hacer arqueología. Así, establecía una secuencia de desarrollo de la arqueología suramericana: tras una primera etapa "sincronista de los cronistas y viajeros occidentales" relativa a los siglos XVI a XIX, durante los últimos 100 años se habría transitado a una etapa de inventario de los más notables vestigios de la cultura indígena, y luego, a una de ordenamiento cronológico y corográfico de las evidencias para llegar, en los últimos años, a una etapa madura de "búsqueda sistemática de una historia que requiere explicación" (Lumbreras ,1992, p. 28). Aun cuando en las primeras etapas reconocía la importancia de la acumulación de datos arqueológicos e históricos, consideraba que la madurez alcanzada, por lo menos desde la década de 1950, se debía a la superación del empirismo para dar paso al desarrollo de una "tradición académica propia", a cargo de arqueólogos suramericanos, formados en sus países o en el extranjero. Pero también registraba un compromiso político con la historia y el conocimiento propios: "No estamos reconstruyendo cualquier historia, sino la nuestra", decía (Lumbreras, 1992, p. 31).

Durante las dos últimas décadas, los planteamientos de Lorenzo y Lumbreras y, más ampliamente, de lo que se ha denominado "el Grupo de Teotihuacán", han sido retomados como referente político y académico de una tradición regional de orientación marxista denominada "arqueología social latinoamericana". Observando desde Filadelfia con interés este movimiento, Patterson (1994, p. 531) lo calificó como "un logro impresionante de los últimos 20 años", que "indica la disposición y capacidad de los académicos de diferentes países para cooperar y colaborar en un mismo proyecto". Desde entonces, un cuerpo importante de literatura se ha producido en la perspectiva de destacar este enfoque, empleando argumentos basados en la historia de la arqueología regional y su relación problemática con las trayectorias europeas y anglosajonas (Benavides, 2001; Fournier, 1999; Jackson, Troncoso y Salazar, 2012; 
McGuire y Navarrete, 1999; Navarrete, 2012; Patterson, 1994, 1997; Tantalean y Aguilar, 2012). A este propósito, Navarrete (2012, p. 55) ha dotado a la arqueología social latinoamericana de una historia propia, ordenada en una secuencia de seis etapas, advirtiendo que ésta no sería aplicable de manera generalizada a todos los contextos nacionales: antecedentes (etapa latente), premarxista (etapa pre-Lima), surgimiento científico-político (etapa Teotihuacán), aplicaciones a sociedades concretas (etapa post-Teotihuacán), refinamiento teórico (etapa Oaxtepec) y énfasis en la teoría crítica (etapa post-Oaxtepec). Se trata de un proceso gradual de adquisición progresiva de conciencia política y producción de una teoría propia.

Una perspectiva opuesta ofrecía Oyuela (1994a) como latinoamericano en Pittsburgh. Criticó la tipología de Trigger (1984) sobre arqueologías nacionales, coloniales e imperiales en cuanto no permitía identificar variaciones locales en los sistemas políticos de los diferentes países, y cómo esas variaciones se relacionan con la práctica de la arqueología. Consideró apropiado diferenciar entre Estado y Nación, entendiendo que el nacionalismo como ideología era una condición para la formación de la Nación (adquisición de una conciencia nacionalista), mientras que el Estado era una condición antecedente. Recurriendo a un modelo derivado de la historia de formación de las naciones europeas (Hobsbawm, 1990; Hroch, 1985), propuso que la historia de la arqueología latinoamericana podría verse como un proceso compuesto por tres fases, advirtiendo que el modelo requería ser sometido a prueba (Oyuela, 1994b, p. 10). Durante la Fase A (proto-estatal), la arqueología no posee ningún apoyo significativo del Estado, como tampoco políticas para la protección de sitios y objetos arqueológicos. Es una práctica a cargo de extranjeros o aficionados locales, con enfoques procesuales entre los primeros, y afines al particularismo cultural entre los segundos. Ponía como ejemplos las arqueologías de Guatemala, Honduras y Belice. En la Fase B (estatal), sectores de clase media con ideologías liberales, logran el apoyo del Estado en la promoción de proyectos de identidad nacionalista. En consecuencia, se generan leyes, políticas y entidades gubernamentales para regular las prácticas relacionadas con lo arqueológico, la formación profesional, y se crean o fortalecen museos nacionales. Los arqueólogos trabajan para el Estado y son financiados por éste, de tal forma que el nivel de desarrollo de la disciplina depende de las condiciones económicas del país. El particularismo histórico cultural es el enfoque más frecuente, dado que resulta funcional a la idea de un origen remoto de la nacionalidad. Ponía como ejemplos México, Perú y Colombia. Finalmente, la Fase C (nacional) se caracteriza por una arqueología con mayor independencia económica e ideológica frente a la estructura política del Estado, creándose fundaciones de investigación y universidades privadas, operándose una descentralización de los museos y una más amplia divulgación del conocimiento arqueológico, el cual se produce desde diferentes enfoques. Para el momento en que escribe Oyuela, sólo en Estados Unidos, Alemania, Francia, Inglaterra y Japón se habría alcanzado esta última fase, cuando "por primera vez la arqueología puede desarrollarse por fuera de constreñimientos políticos a favor de una arqueología más científica o académica" (Oyuela, 1994b, p. 16).

Este esquema se encuentra estructurado por una teleología evolutiva que transcurre desde un estadio pre-científico a uno de carácter científico, de tal manera que distintas fases, más o menos avanzadas en el camino de una arqueología verdaderamente científica, coexisten en diferentes partes del mundo. En este sentido, el valor de comprender la historia de la arqueología en Latinoamérica, estribaría en la identificación de externalidades en las fases A y B, que deberían ser erradicadas para alcanzar, en la fase $\mathrm{C}$, una producción arqueológica eminentemente científica, al estilo norteamericano, europeo y japonés. De ahí que en años posteriores haya criticado la apuesta por una arqueología social latinoamericana. Con otros autores (Oyuela, Anaya, Elera y Valdez, 1997), argumentó, en contra de lo planteado por Patterson (1994), que su desarrollo estuvo limitado a ciertos países (Chile, México, Perú y Venezuela), que su 
expansión e impacto fueron débiles en el espacio y el tiempo y que, además, reprodujo esquemas conceptuales no propiamente marxistas, como el enfoque histórico-cultural (ver Patterson, 1997, para una réplica).

En medio de esta tensión, los trabajos efectuados por Politis desde La Plata, permiten identificar matices en las relaciones entre las trayectorias locales de las arqueologías latinoamericanas y los enfoques desarrollados en Norteamérica y Europa. El autor explora estas relaciones en términos de "tradiciones" e "influencias". En una primera aproximación señaló que "no existe una 'arqueología latinoamericana' teóricamente homogénea y metodológicamente compacta, así como tampoco parece existir una corriente hegemónica", si no que coexisten diferentes enfoques y programas de investigación resultado de "un complejo proceso que incluye las tradiciones en investigaciones nacionales o regionales y las influencias teóricas y metodológicas que operaron en cada país" (Politis, 1992, p. 14). Consideraba, sin embargo, que estas arqueologías se habían caracterizado por el empirismo y la ausencia de producción teórica, con lo cual los arqueólogos latinoamericanos serían "en su inmensa mayoría lectores e 'importadores' de teorías" y "usuarios (en el mejor de los casos adaptadores) de métodos generados fuera de la región" (Politis, 1992, p. 27). Posteriormente, matizó esta aseveración:

(...) sería injusto ver la arqueología latinoamericana como un reflejo pasivo de influencias foráneas, fundamentalmente norteamericanas. Ha habido seguramente arqueólogos locales que han desarrollado métodos originales, aun cuando obviamente sus contribuciones se han alimentado de teorías y métodos foráneos (Politis, 1999, p. 4).

Propuso entonces un esquema básico de sucesión y coexistencia de "influencias teóricas" foráneas en las tradiciones locales, cuya importancia habría fluctuado según la geopolítica internacional. Inicialmente (siglo XIX) habrían primado ideas evolucionistas europeas, pero luego serían los enfoques difusionistas e historicistas los más importantes, derivados de teorías elaboradas en Norteamérica, Inglaterra, Alemania y Austria. Luego de la Segunda Guerra Mundial, la influencia norteamericana creció notablemente dando como resultado, bien el fortalecimiento de los enfoques histórico-culturales, o más tarde, el desarrollo de enfoques ecológico-sistémicos ligados al procesualismo, aunque los primeros seguirían predominando. A partir de la década de 1970, el panorama se habría diversificado al coexistir los anteriores enfoques con perspectivas marxistas, como la arqueología social latinoamericana, planteamientos proclives al posprocesualismo británico y algunos desarrollos ligados al estructuralismo y posestructuralismo franceses y al neoevolucionismo norteamericano (Politis 1995, 2003).

Preocupaban especialmente a Politis dos aspectos que también serían enfatizados en la síntesis efectuada luego por Dillehay (2008): el poco interés de los arqueólogos latinoamericanos por la teoría y la poca visibilidad internacional de su producción. Se trataba de una condición doblemente periférica que mantenía más o menos aislados a los arqueólogos latinoamericanos. En opinión del autor, esta situación distaba de la simple oposición entre arqueólogos "gringos imperialistas" y "pobrecitos latinoamericanos", obedeciendo más bien a "una serie compleja de relaciones históricas, políticas y económicas entre Latinoamérica, las naciones más industrializadas de Europa y los Estados Unidos" (Politis, 2003, p. 131). La solución estaría en un esfuerzo mutuo de los arqueólogos latinoamericanos por alcanzar un nivel más alto de producción teórica y metodológica y de los colegas extranjeros, por incorporar dichas producciones en el ámbito global de la arqueología.

Esta noble tarea no sería fácil, a la luz de una "economía política de la arqueología", planteada por Gnecco (2009, p. 241) desde Popayán. Con un enfoque decolonial, 
ha descrito la historia de las "arqueologías latinoamericanas" como un proceso de producción de discursos sobre el pasado funcionales al colonialismo de los proyectos modernos y nacionales, primero, y multiculturalistas en las últimas décadas. Identifica una "geopolítica del conocimiento" (en el sentido de Mignolo, 2002), tanto en las relaciones asimétricas de poder entre las arqueologías metropolitanas y latinoamericanas, como entre éstas últimas y formas de la memoria y la historia producidas por actores subalternos. Esta manera de aproximarse a la historia de la arqueología regional soportó, en primera instancia, la apuesta por una "arqueología multivocal" (Gnecco, 1999), luego por una "arqueología de la interculturalidad y la descolonización” (Gnecco, 2009, p. 263) y, últimamente, por "alternativas a la arqueología” e incluso por unas "no-arqueologías" (Gnecco, 2017, p. 155).

Por supuesto que en ese trayecto fueron quedando cancelados los caminos hacia una arqueología orientada por modelos científicos de alcance global, mientras que las aspiraciones de producir una "arqueología propia" no tendrían como referente la arqueología marxista latinoamericana que “(...) no fue original (sólo la repetición de un canon ortodoxo e inflexible), no rescató un pensamiento "propio" (cuyo sentido, en todo caso, es equívoco) y su apuesta política fue, por lo menos, marginal" (Gnecco, 2009, p. 252). Finalmente, quedaron canceladas la "arqueología multicultural" que, en última instancia, domestica la alteridad y sirve a una mercantilización de la diferencia cultural, así como las arqueologías "públicas" e incluso “indígenas”, por cuanto reproducirían perversamente el proyecto ilustrado y moderno de una arqueología disciplinar que se expande (Gnecco, 2017). En el enfoque de Gnecco, estudiar y narrar las historias de la arqueología, lejos de ser un ejercicio edificante, tiene como objetivo fundamentar la crítica radical de su estructura disciplinar y de su profunda relación con el proyecto moderno. En este sentido, la espacialidad a la que podría remitir una historia de la arqueología como dispositivo de la colonialidad del poder ya no se limitaría a Latinoamérica, sino que sería cuestión del "sur global” (Shepherd, Gnecco y Haber, 2016).

Al examinar planteamientos tan diversos, se puede decir que el carácter específico de las arqueologías latinoamericanas y por extensión suramericanas e incluso iberoamericanas, no es necesariamente una cuestión deseable o condenable, sino el resultado concreto de dinámicas geopolíticas y particulares factores económicos, políticos e ideológicos que, en su conjunción, definen condiciones de posibilidad para lo que son y pueden ser hacia el futuro las prácticas de la arqueología en la región. Adicionalmente, y de acuerdo a lo planteado por Lumbreras (1992), es necesario no dejar de lado la particularidad que ofrecen las evidencias arqueológicas y registros documentales de la región. Pero este hecho no implica automáticamente una particularidad en la forma en que se ha practicado la arqueología en la región y mucho menos, una manera singular de hacer la historia de esa práctica. No hay una correlación "natural" y dada entre estas esferas (evidencias, praxis e historia de la praxis), porque las categorías mismas de Latinoamérica, Suramérica o Iberoamérica, son producciones geohistóricas, es decir, han emergido y se han transformado en relación con específicos regímenes espaciales e históricos ${ }^{2}$.

La visibilidad y sentido de la arqueología latinoamericana dependen de dinámicas simultáneas de territorialización-desterritorialización-reterritorialización ${ }^{3}$ que encuentran en las historias de la arqueología un dispositivo fundamental. Mientras las historias nacionales y regionales de la arqueología, territorializan determinados espacios para demarcar el carácter propio o periférico, las historias orientadas por un sentido global de la arqueología los desterritorializan, como condición de posibilidad de una ciencia válida en cualquier parte. En cuanto a las historias poscoloniales o decoloniales, efectúan una reterritorialización basada, no ya en espacios nacionales o subcontinentes, sino en espacios menos contiguos, más bien archipiélagos, en donde se inscriben relaciones coloniales.
2. Se emplea aquí el concepto de geohistoria en el sentido definido por Soja (2010, p. 362): “(...) la geohistoria representa mucho más que agregar una dosis extra de geografía a la escritura de la historia, o simplemente reconocer que 'el espacio importa'. Como lo veo, geohistoria es indicativa de una forma radicalmente diferente de mirar la historia y la geografía juntas, tratándolas como mutuamente formativas y equivalentes en su poder interpretativo".

3. En el mismo sentido en que Deleuze y Guattari (1997, p. 86), en su propuesta de una geofilosofía, emplean estos conceptos para plantear distintos movimientos por los cuales el pensamiento filosófico se relaciona de maneras diferentes con los planos de inmanencia, los suelos, la Tierra. 


\section{Conclusiones}

Es un asunto advertido en la literatura especializada, que las historias de la arqueología han sido escritas fundamentalmente por arqueólogos y para arqueólogos (Christenson, 1989, p. 1; Díaz, 2007, p. 1; Trigger, 2001, p. 630), resaltando el género masculino (Díaz y Sørensen, 1998). Y son pocos los estudios efectuados desde otros campos del saber o en relación con problemáticas más amplias que la arqueología misma (Embree, 1987; Moro, 2012; Podgorny, 2009; Salmon, 1982; Wylie, 2002). Incluso desde las historias de la antropología y la etnología, tan cercanas a la arqueología, han sido débiles los acercamientos a ésta última (Harris, 1978; Lowie, 1985).

Los arqueólogos han escrito la historia de su quehacer contando, básicamente, con el repertorio de conceptos y estrategias analíticas que suelen tener a su alcance para estudiar los vestigios que constituyen su objeto de estudio. Así, en sus historias hay cierta predilección por el establecimiento de "etapas" y "fases"; se suelen describir diferentes enfoques teórico-metodológicos en términos de "tradiciones" ligadas a específicos territorios, a menudo nacionales; y se habla de "influencias" teórico-metodológicas, que parecerían comportarse como fenómenos de migración y difusión cultural. En este sentido, puede decirse que, en su introspección, los arqueólogos han "arqueologizado" la historia de su praxis.

Buena parte de las conexiones que las historias de la arqueología han tenido con concepciones más amplias sobre el devenir de las ciencias, se ha hecho a través de la apropiación de enfoques aplicados al ejercicio sustantivo de la disciplina (Trigger, 2001). Por ejemplo, mientras una parte importante de la arqueología del siglo XIX e inicios del XX se edificó en torno a la descripción de vestigios monumentales, algunas de las primeras historias disciplinares exaltaron la figura de prominentes "descubridores" de esos monumentos, como es evidente en la célebre novela histórica de Ceram (1985), pero también en otros textos (Casson, 1940; Daniel, 1950; Michaelis, 1908). Encontraban aquí, por la vía de los grandes "precursores" y sus espectaculares hallazgos, el vínculo profundo con una historia heroica y monumental de la ciencia. Posteriormente, el compromiso con la agenda de una epistemología positivista y la adopción de enfoques neoevolucionistas, propios de la arqueología procesual o Nueva Arqueología, fue afín a perspectivas de la ciencia como una empresa que avanza gracias a la innovación y el rigor de la técnicas, métodos y teorías, minimizando o dejando de lado los aspectos extra-académicos, que, en todo caso, se consideran como externalidades que no deben incidir en la ciencia (Willey y Sabloff, 1974). Por otra parte, desde las arqueologías posprocesuales o interpretativas, la incorporación de conceptos de la historia social y las teorías del poder en pro de la comprensión del registro arqueológico, se trasladó a los esfuerzos para comprender el devenir mismo de la disciplina, produciéndose algunas de las primeras "historias sociales de la arqueología" (Trigger, 1992). Así mismo, el propósito de visibilizar a las mujeres y las diferencias de género en el pasado que estudia la disciplina (Gilchrist, 1999, p. 4), se ha proyectado a los ejercicios mismos de historización de la arqueología (Claassen, 1994; Díaz y Sørensen, 1998). Finalmente, el interés de los enfoques marxistas por analizar la relación entre determinadas formaciones económico-sociales y organizaciones políticas en las sociedades pretéritas, se encuentra también en las narrativas de la historia disciplinar (Patterson, 1995).

En años más recientes, se observa una cierta transformación en estas conexiones, cuando se han incorporado planteamientos derivados de la historia y los estudios de la ciencia, lo cual ha llevado a identificar ciertos "desfases" teóricos y metodológicos en la manera en que se venían narrado las historias de la arqueología (Kaeser, 2002, 2008; Moro, 2009, 2010, 2012; Murray, 2002; Schlanger, 2002). Así mismo, se nota una apropiación más inmediata de enunciados provenientes de enfoques poscoloniales o decoloniales que denuncian el proyecto de la ciencia como una práctica hegemónica, 
androcéntrica, eurocentrada y colonial (Gnecco, 2017; Gosden, 2004). Además, se ha propuesto la necesidad de hacer visibles, en las historias de la arqueología, a todos aquellos sujetos que han desempeñado roles subalternos en las investigaciones como auxiliares, ayudantes, colaboradores y trabajadores (Kehoe, 1998, p. ix). Y han comenzado a escribirse historias que hacen visibles tensiones entre las prácticas arqueológicas y las concepciones de los pueblos indígenas, no sólo por parte de los académicos (Atalay, 2006; Gnecco y Ayala, 2010), sino de autores indígenas (Mamani, 1996; Riding In, 1992; Watkins, 2005). Al fin y al cabo, la arqueología y sus historias no pertenecen sólo a los arqueólogos (Murray, 2002, p. 238).

En ese sentido cabe decir, como Murray y Evans (2008, p. 2), que "las grandes metanarrativas de la historia de la arqueología" vienen siendo desafiadas por la historiografía más reciente de la disciplina. Pero el ejercicio de espacialización de las historias de la arqueología que se ha propuesto en este artículo, sugiere que en muchas de ellas aún hace carrera un imaginario espacio-temporal, según el cual, el tronco principal de la arqueología tiene sus raíces en el pensamiento científico de Europa occidental y del mundo anglosajón, siendo las demás trayectorias ramas más o menos débiles, mediante las cuales se ha extendido la savia de una arqueología ya hecha. En este proceso de "difusión", las distancias geográficas pero, sobre todo, las condiciones económicas, políticas y culturales locales, además de constituir obstáculos a vencer en pro de una arqueología científica de talla mundial, explicarían las imperfecciones y retrasos en la adopción de los modelos europeos y/o anglosajones, cuyos rasgos precoces y actuales de madurez, son tomados como paradigmas a seguir. Este esquema "arbóreo" (Murray y Evans, 2008, p. 1), prevalece incluso en los análisis historiográficos propiamente dichos. La celebrada idea de una mayoría de edad alcanzada por el campo de la historia de la arqueología, corresponde a una metáfora orgánica que asimila el devenir del conocimiento arqueológico al del ciclo vital de crecimiento y de obtención de autoridad política de los individuos en Occidente. La mayoría de edad supone el haber transitado desde una condición de inocencia y dependencia (pre-científica) a una de plena conciencia de sí y autonomía (científica), lo que en términos disciplinares significa haber producido, innovado y acumulado con suficiencia enfoques, metodologías, técnicas y datos.

Se trata de una metáfora que resulta sintomática de la prevalencia de unos regímenes históricos y espaciales que escapan a buena parte de los ejercicios reflexivos y críticos que supone hacer una historiografía. Estos regímenes, además de reproducir una concepción teleológica de la ciencia, operan con base en algunas categorías que son propias de una imaginación geográfica de la modernidad, la cual ha incidido en las geografías del conocimiento (Agnew, 2003, 2007). En primer lugar, el mundo como totalidad estaría compuesto fundamentalmente por espacios nacionales que fungen como las unidades básicas que por excelencia sirven al "recorte" de las dinámicas que interesan a los historiadores de la arqueología. En segundo lugar, las diferencias espaciales se ordenan temporalmente, de tal forma que la arqueología efectuada en y desde los "centros" tiene más historia y es científicamente más avanzada que la efectuada en las periferias, siendo incluso referentes para el futuro. Algo que se pone de manifiesto en el lugar vacío o secundario que a menudo han ocupado las arqueologías latinoamericanas en las narrativas "mundiales" de historia de la arqueología.

Es cierto que la centralidad de los espacios nacionales no es exclusiva de la historiografía de la arqueología, sino que constituye un rasgo frecuente de las historias de la ciencia (Livingstone, 2003, p. 123). También que la arqueología ofrece casos emblemáticos de uso político del pasado por parte de los proyectos nacionales (Arnold, 1996; Díaz, 2010; Fowler, 1987; Klejn, 1993; Kohl, 1998). Pero las historias de la ciencia centradas en los espacios nacionales, e incluso aquellas que apelan a regiones o subregiones como simples agregados de estados nacionales, además de contribuir con una naturalización 
de los territorios estatales en la geopolítica actual, pueden ocultar dinámicas diversas que acontecen al interior de lo nacional, o que atraviesan las fronteras entre los países (Evans, 2008; Lozny, 2011b).

Ampliamente, una geografía de las ciencias y para el caso, de la arqueología, debe comprender "no sólo cómo el conocimiento es producido en lugares específicos, sino también, cómo acontecen las transacciones entre esos lugares" (Shapin, 1998, pp. 6-7). De tal forma que debe ser sensible a las muy diferentes espacialidades que intervienen en la manera en que el conocimiento se genera, se moviliza y se apropia, se reproduce o se niega: cuerpos, sitios, arquitecturas, lugares, regiones, territorios, fronteras, circuitos y redes, configuran esa constelación de geografías que no siempre funcionan de manera escalar. Tal como lo plantea Finnegan: la ciencia es

(...) un conglomerado dinámico de prácticas, materialidades y personas, diferencialmente ensamblados en distintos lugares, y basados en la traducción y transformación - más que en una simple difusión- de datos y teorías. Las implicaciones de esto son que lo local y lo regional, no son puntos fijos o territorios limitados, sino más bien instancias de redes y circuitos más amplios (Finnegan, 2008, p. 38).

De ahí la inconveniencia de un esquema arbóreo de la historia de la arqueología, que invisibiliza, o cuando más, integra las "periferias" como débiles extensiones de una historia ya hecha en los centros. Comprender las diferentes trayectorias de la arqueología, incluso aquellas situadas en Latinoamérica, África, Asia y Oceanía, es imprescindible para entender cómo se han hecho y narrado las arqueologías en Europa y Norteamérica. Si las historias de la arqueología pueden contribuir a comprender los procesos y tendencias que deben ser tenidos en cuenta a la hora de trazar su ejercicio futuro, las geografías de la arqueología pueden suministrar coordenadas indispensables para la generación de conocimientos que no se conforman con saberse localizados, sino que aspiran a dialogar desde una perspectiva situada (Haraway, 1988) del conocimiento.

\section{Agradecimientos}

Este texto se deriva en primera instancia del proceso de formación doctoral en Historia de la Universidad de los Andes, siendo re elaborado posteriormente en desempeño de mis labores como investigador y docente del Instituto de Estudios Regionales de la Universidad de Antioquia y de su Grupo Estudios del Territorio. Gracias a quienes en esas instituciones, Mauricio Nieto, Carl Langebaek y Vladimir Montoya, me apoyaron con sus lecturas, comentarios y el tiempo necesario para la escritura. Así mismo, doy mi agradecimiento a los evaluadores anónimos designados por la revista Arqueología por los aportes realizados sobre el manuscrito original. 


\section{Referencias citadas}

》Agnew, J. (2003). Geopolitics, re-visioning Worlds politics. Londres: Routdledge.

"Agnew, J. (2007). Know-Where: Geographies of Knowledge of World Politics. International Political Sociology, 1, 138-148.

" Arnold, B. (1996). The Past as Propaganda: Totalitarian Archaeology in Nazi Germany. En R. Preucel y l. Hodder (Eds.), Contemporary Archaeology in Theory. A Reader (pp. 549569). Oxford: Blackwell Publishing.

»Atalay, S. (2006). Indigenous Archaeology as Decolonizing Practice. The American Indian Quarterly, 30(3-4), 280-310.

» Bahn, P. (Ed.). (1996). Cambridge illustrated history of archaeology. Cambridge: Cambridge University Press.

» Bahn, P. (2014). The History of archaeology. An introduction. Oxon: Routledge.

》Bernal, I. (1979). Historia de la arqueología en México. México: Editorial Porrúa.

"Benavides, H. (2001). Returning to the source: Social archaeology as Latin American philosophy. Latin American Antiquity, 12(4), 355-370.

" Boast, R. (2009). The Formative Century, 1860-1960. En B. Cunliffe, Ch. Gosden y R. Joyce (Eds.), The Oxford Handbook of Archaeology (pp. 55-70). Toronto: Oxford University Press.

" Casson, S. (1940). The discovery of man. The story of the inquiry into human origins. Londres: Readers Union Limited.

"Ceram, W. (1985). Dioses, Tumbas y Sabios. Barcelona: Ediciones Orbis.

"Christenson, A. (1989). Preface. En A. Christenson (Ed.), Tracing Archaeology's past: The Historiography of Archaeology (pp. ix-xi). Carbondale: Southern Illinois University Press.

»Claassen, C. (1994). Introduction. En C. Claassen (Ed.), Women in Archaeology (pp. 1-8). Filadelphia: University of Pennsylvania Press.

" Corbey, R. y Roebroeks, W. (2001). Studying Human Origins. Disciplinary History and Epistemology. Amsterdam: Amsterdam University Press.

"Cunliffe, B., Gosden, C. y Joyce, R. (Eds.). (2009). The Oxford Handbook of Archaeology. Toronto: Oxford University Press.

》Curtoni, R. y Politis, G. (2006). Race and racism in South American archaeology. World Archaeology, 38(1), 93-108.

» Cyphers, A. (2014). Mesoamerica. En P. Bahn (Ed.), The History of archaeology. An introduction (pp. 194-209). Abingdon-Nueva York: Routledge.

"Daniel, G. (1950). A hundred years of archaeology. Londres: Duckworth.

"Daniel, G. (1963). The Idea of Prehistory. Cleveland: World Publication Company.

"Daniel, G. (Ed.) (1981). Towards a History of Archaeology. Londres: Thames and Hudson.

" D’Agostino, M. (Ed.) (1999). Anais da Reuniao Internacional de Teoria Arqueológica na América do Sul. San Pablo: Universidad de Sao Paulo. Revista do Museu de Arqueología e Etnologia Suplemento 3.

»Deleuze, G. y Guattari, F. (1997) ¿Qué es la filosofía? Barcelona: Anagrama. 
"Díaz, M. (2007). A World History of Nineteenth-century Archaeology: Nationalism, Colonialism, and the Past. Oxford: Oxford University Press.

"Díaz, M. (2010). Nationalism and Archaeology. Spanish Archaeology in the Europe of Nationalities. En R. Preucel y S. Mrozowksi (Eds.), Contemporary Archaeology in Theory and Practice (pp. 432-444). Londres: Blackwell.

» Díaz, M. y Sørensen, M. (1998). Excavating Women. Towards an engendered history of archaeology. En M. Díaz y M. Sørensen (Eds.), Excavating Women: History of Women in European Archaeology (pp. 1-28). Londres: Routledge.

»Dillehay, T. (2008). Latin American Archaeology in History and Practice. En A. Bentley, H. Maschner y C. Chippindale (Eds.), Handbook of Archaeological Theories (pp. 165-185). Lanham: Altamira Press.

"Duque, L. (1955). Colombia. Monumentos históricos y arqueológicos. Libro primero: Monumentos y objetos arqueológicos. México: Instituto Panamericano de Geografía e Historia.

"Embree, L. (1987). Archaeology: the most basic science of all. Antiquity, 61(231), 75-78.

"Embree, L. (1993). Selected Bibliography of Archaeological Metaarchaeology. En L. Embree (Ed.) Metaarchaeology. Reflections by Archaeologists and Philosophers (pp. 289317). Boston: Boston Studies in the Philosophy and History of Science, Vol. 147, Kluwer Academic Publishers.

»Evans, C. (2008.) Archaeology against the State: Roots of Internationalism. En T. Murray y C. Evans (Eds.), Histories of Archaeology. A Reader in the History of Archaeology (pp. 222237). Oxford: Oxford University Press.

"Fernández, J. (1979). Historia de la Arqueología Argentina. Anales de Arqueología y Etnología, XXXIV-XXXV. Mendoza: Universidad Nacional de Cuyo.

" Finnegan, D. (2008). The Spatial Turn: Geographical Approaches in the History of Science. Journal of the History of Biology, 41, 369-388.

" Fournier, P. (1999). La arqueología social latinoamericana: caracterización de una posición teórica marxista. En A. Zarankin y F. Acuto (Eds.), Sed Non Satiata. Teoría social en la arqueología latinoamericana contemporánea (pp. 17-32). Buenos Aires: Tridente.

" Fowler, D. (1987). Uses of the past: Archaeology in the Service of the State. American Antiquity, 52(2), 229-248.

" Funari, P. (1995). Mixed features of archaeological theory in Brazil. En P. Ucko (Ed.), Theory in archaeology. A World Perspective (pp. 232-246). Londres y Nueva York: Rouletdge.

» Funari, P. (2008). A History of archaeology in Brazil (2001). En T. Murray y C. Evans (Eds.), Histories of Archaeology. A Reader in the History of Archaeology, (pp. 328-345). Oxford: Oxford University Press.

» Funari, P., Zarankin, A. y Stovel, E. (Eds.). (2005). Global Archaeological Theory: Contextual Voices and Contemporary Thoughts. Nueva York: Kluwer Academic/Plenum Publishers.

" Funari, P., Zarankin, A. y Stovel, E. (2009). South American Archaeology. En B. Cunliffe, C. Gosden y R. Joyce (Eds.), The Oxford Handbook of Archaeology (pp. 679-704). Toronto: Oxford University Press.

" Fung, R. (1965). Arqueología, ciencia histórica. Un ensayo crítico de los métodos y las teorías en la arqueología peruana. Lima: Universidad Nacional Mayor de San Marcos.

" Gilchrist, R. (1999). Gender and archaeology: contesting the past. Londres: Routledge.

"Gnecco, C. (1999). Multivocalidad histórica. Hacia una cartografía poscolonial de la arqueología. Bogotá: Universidad de los Andes. 
"Gnecco, C. (2009). Arqueologías latinoamericanas: de la modernidad a los Estados multiculturales. Cadernos de Ciências Humanas, 11-12(20-21), 241-271.

» Gnecco, C. (2017). Antidecálogo. Diez ensayos (casi) arqueológicos. Popayán: Universidad del Cauca.

» Gnecco, C. y Ayala P. (Comps.). (2010). Pueblos indígenas y arqueología en América Latina. Bogotá: Banco de la República - Universidad de los Andes.

» Gosden, C. (2004). The Past and Foreign Countries: Colonial and Post-Colonial Archaeology and Anthropology. En L. Meskell y R. Preucel (Eds.), A Companion to Social Archaeology (pp. 161-178). Oxford: Blackwell.

» Gregory, D. (2009). Geographical imagination. En G. Derek, R. Johnston, G. Pratt, M. Watts y S. Whatmore (Eds.), Dictionary of Human Geography (pp. 282-285). Chichester: Wiley-Blackwell.

» Haber, A. (2014). South American Archaeology: Postcolonial Perspectives. En C. Smith (Ed.), Encyclopedia of Global Archaeology (pp. 6822-6828). Nueva York: Springer Science - Business Media.

» Haber, A. y Roberts, A. (2014). Histories of the Archaeological Discipline: Issues to Consider. En C. Smith (Ed.), Encyclopedia of Global Archaeology (pp. 3411-3415). Nueva York: Springer Science - Business Media.

» Haraway, D. (1988). Situated Knowledges: The Science Question in Feminism and the Privilege of Partial Perspective. Feminist Studies, 14(3), 575-599.

» Harris, M. (1978). El desarrollo de la teoría antropológica. Historia de las teorías de la cultura. Madrid: Siglo XXI.

» Hartog, F. (2007). Regímenes de historicidad, presentismo y experiencias del tiempo. México: Universidad Iberoamericana.

»Hegmon, M. (2003). Setting Theoretical Egos Aside: Issues and Theory in North American Archaeology. American Antiquity, 68(2), 213-243.

» Herrera, A. (Comp.). (2013). Arqueología y desarrollo en América del Sur: de la práctica a la teoría. Bogotá: Universidad de los Andes.

» Hobsbawm, E. (1990). Nations and Nationalism since 1780. Programme, myth, reality. Cambridge: Cambridge University Press.

»Hroch, M. (1985). Social preconditions of national revival in Europe: a comparative analysis of the social composition of patriotic groups among the smaller European nations. Cambridge: Cambridge University Press.

» Jackson, D., Troncoso, A. y Salazar, D. (2012). Hacia una crítica de la práctica de la arqueología social latinoamericana. En H. Tantalean y M. Aguilar (Comps.), La Arqueología Social Latinoamericana: de la teoría a la praxis (pp. 49-62). Bogotá: Universidad de los Andes.

》 Jaramillo, L. (Ed.). (2008). Arqueología en Latinoamérica: historias, formación académica y perspectivas temáticas. Bogotá: Universidad de Los Andes.

» Johnson, M. (2009). The Theoretical Scene, 1960-2000. En B. Cunliffe, Ch. Gosden y R. Joyce (Eds.), The Oxford Handbook of Archaeology (pp. 71-82). Toronto: Oxford University Press.

» Kaeser, M. (2002). On the international roots of prehistory. Antiquity, 76(291), 170-177.

» Kaeser, M. (2008). Biography as Microhistory: The Relevance of Private Archives for Writing the History of Archaeology. En N. Schlanger y J. Nordblach (Eds.), Archives, Ancestors, Practices. Archaeology in the Light of its History (pp. 9-20). Nueva York: Bergham Books. 
" Kehoe, A. (1998). The Land of Prehistory: A Critical History of American Archaeology. Nueva York: Routledge.

» Klejn, L. (1993). La arqueología soviética. Historia y teoría de una escuela desconocida. Barcelona: Editorial Crítica.

» Kohl, P. (1998). Nationalism and Archaeology: On the Constructions of Nations and the Reconstructions of the Remote Past. Annual Review of Anthropology, 27, 223-246

» Kuhn, T. (1970). The structure of Scientific Revolutions. Chicago: The University of Chicago Press.

» Lepenies, W. y Weingar, P. (1983). Introduction. En L. Graham, W. Lepenies y P. Weingart (Eds.), Functions and Uses of Disciplinary Histories (pp. ix-xx). Dordrecht: D. Reidel Publishing Co.

》 Livingstone, D. (2003). Putting Science in its place: geographies of Scientific Knowledge. Chicago: The University of Chicago Press.

" Lopez, E. (2014). South America. En P. Bahn (Ed.), The History of archaeology. An introduction (pp. 210-227). Abingdon-Nueva York: Routledge.

» Lorenzo, J. (1981a). Archaeology South of the Rio Grande. World Archaeology, 13(2), 190-208.

» Lorenzo, J. (1981b). Notes on the history of Ibero-American archaeology. En G. Daniel (Ed.) Towards a History of Archaeology (pp. 133-145). Londres: Thames and Hudson.

» Lowie, R. (1985). Historia de la Etnología. México: Fondo de Cultura Económica.

» Lozny, L. (Ed.). (2011a). Comparative archaeologies. A sociological view of the science of the past. Nueva York: Springer

» Lozny, L. (2011b). Archaeology in the Age of Globalization: Local Meanings, Global Interest. En L. Lozny (Ed.), Comparative archaeologies. A sociological view of the science of the past (pp. 21-49).Nueva York: Springer.

" Lumbreras, L. (1992). La arqueología sudamericana: tres décadas. En B. Meggers (Ed.), Prehistoria sudamericana: nuevas perspectivas (pp. 27-32).Washington: Taraxacum.

» Mamani, C. (1996). History and prehistory in Bolivia: What about the Indians, En R. Preucel y I. Hodder (Eds.), Contemporary archaeology in theory (pp. 632-645). Oxford: Blackwell Publishers.

" Martínez, A., Taboada, C. y Auat, A. (2008). The Wagner Brothers: French Archaeologists and Original Myths in Early Twentieth Century Argentina. En N. Schlanger y J. Nordblach (Eds.), Archives, Ancestors, Practices. Archaeology in the Light of its History (pp. 261-271). Nueva York: Bergham Books.

» McGuire, R. y Navarrete R. (1999). Entre motocicletas y fusiles: las arqueologías radicales Anglosajona y Latinoamericana. Boletín de Antropología Americana, 34, 89-110.

» Meltzer, D. (1989). A question of relevance. En A. Christenson (Ed.), Tracing Archaeology's past: The Historiography of Archaeology (pp. 5-20). Carbondale: Southern Illinois University Press.

» Meskell, L. y Preucel, R. (Eds.) (2004). A companion to social archaeology (pp. 353-373). Oxford: Wiley.

" Michaelis, A. (1908). A Century of Archaeological Discoveries. Londres: John Murray.

» Mignolo, W. (2002). The geopolitics of knowledge and the colonial difference. South Atlantic Quarterly, 101(1), 56-96.

»Moro, O. (2006). The History of Archaeology as a 'Colonial Discourse'. Bulletin of the History of Archaeology, 16(2), 4-17. 
" Moro, O. (2009). The History of Archaeology as Seen Through the ExternalismInternalism Debate: Historical Development and Current Challenges. Bulletin of the History of Archaeology, 19(2), 13-26.

» Moro, O. (2010). Beyond externalism. Exploring new directions in the history of archaeology. Archaeological Dialogues, 17(2), 215-236.

» Moro, O. (2012). La nueva historia de la arqueología: Un balance crítico. Complutum, 23(2), 177-190.

» Moro, O. (2013). The History of Archaeology as a Field: From Marginality to Recognition. En S. Chrisomalis y A. Costopoulos (Eds.), Human Expeditions: Inspired by Bruce Trigger (pp. 90-101). Toronto: University of Toronto Press.

» Murray, T. (Ed.). (1999). Encyclopedia of Archaeology: The Great Archaeologists (2 Volumes). Santa Barbara: ABC-CLIO Press.

» Murray, T. (2001). Encyclopedia of Archaeology: History and Discoveries. Santa Barbara: ABC-CLIO Press.

» Murray, T. (2002). Epilogue: why the history of archaeology matters. Antiquity, 76(291), 234-238.

» Murray, T. (Ed.) (2007a). Milestones in Archaeology: A Chronological Encyclopedia. Santa Barbara; ABC-CLIO Press.

» Murray, T. (2007b). Introduction. En T. Murray (Ed.). Milestones in Archaeology: A Chronological Encyclopedia, (pp. xiii-xxv). Santa Barbara: ABC-CLIO Press.

» Murray, T. y Evans, Ch. (Eds.) (2008). Histories of Archaeology. A Reader in the History of Archaeology. Oxford: Oxford University Press.

» Nastri, J. y Menezes, L. (Eds.) (2010). Historias de Arqueología Sudamericana. Buenos Aires: Fundación de Historia Natural Félix de Azara.

» Navarrete, R. (2012). ¿El fin de la Arqueología Social latinoamericana? Reflexiones sobre la trascendencia histórica del pensamiento marxista sobre el pasado desde la geopolítica del conocimiento latinoamericano. En H. Tantalean y M. Aguilar (Comps.), La Arqueología Social Latinoamericana: de la teoría a la praxis (pp. 45-66). Bogotá: Universidad de los Andes.

»Ophir, A. y Shapin, S. (1991). The Place of Knowledge. A Methodological Survey. Science in Context, 4(1), 3-22.

» Oyuela, A. (Ed.). (1994a). History of Latin American Archaeology. Aldershot: Ashgate Publishing Company.

» Oyuela, A. (1994b). Nationalism and Archaeology: A Theoretical Perspective. En A. Oyuela (Ed.), History of Latin American Archaeology (pp. 3-21). Aldershot: Ashgate Publishing Company.

» Oyuela, A., Anaya, A., Elera, C. y Valdez, L. (1997). Social Archaeology in Latin America?: Comments to T. C. Patterson. American Antiquity, 62(2), 365-374.

»Patterson, Th. (1994). Social Archaeology in Latin America: An Appreciation”. American Antiquity, 59(3), 531-537.

» Patterson, Th. (1995) Towards a social history of archaeology in the United States. Forth Worth: Harcourt Brace \& Company.

» Patterson, T. (1997). A Reply to A. Oyuela-Caycedo, A. Anaya, C. G. Elera, y L. M. Valdez. American Antiquity, 62(2), 375-376.

» Piazzini Suárez, C. (2010). Geografías del conocimiento: transformación de los protocolos de investigación en las arqueologías latinoamericanas. Geopolítica(s), 1(1), 115-136. 
"Podgorny, I. (2009). El Sendero del tiempo y de las causas accidentales. Los espacios de la prehistoria en la Argentina. Rosario: Protohistoria Ediciones.

»Politis, G. (1992). Introducción. En G. Politis (Ed.), Arqueología en América Latina Hoy (pp. 14-29). Bogotá: Banco Popular.

" Politis, G. (1995). The socio-politics of the development of archaeology in Hispanic South America. En P. Ucko (Ed.), Theory in archaeology. A World Perspective (pp. 194-231). Londres y Nueva York: Rouletdge.

» Politis, G. (1999). Introduction. Latin American archaeology: an inside view. En G. Politis y B. Alberti (Eds.), Archaeology in Latina America (pp. 1-14). Londres y Nueva York: Routledge.

"Politis, G. (2003). The theoretical landscape and the methodological development of archaeology in Latin America. American Antiquity, 68(2), 245-272.

»Politis, G. y Alberti, B. (Eds.). (1999). Archaeology in Latin America. Londres y Nueva York: Rouletdge.

»Politis, G. y Pérez, J. (2004). Latin American archaeology: between colonialism and globalization. En L. Meskell y R. Preucel (Eds.), A companion to social archaeology (pp. 353-373). Oxford: Wiley.

»Preucel, R. y Hodder, I. (1996). Prologue. En R. Preucel y l. Hodder (Eds.), Contemporary archaeology in theory. A reader (pp. 3-20). Londres: Blackwell.

» Riding In, J. (1992). Without ethics and morality: a historical overview of imperial archaeology and American Indians. Arizona State Law Journal, 24, 11-34.

» Ruiz, G. (2017). Historias generales de la Arqueología. En M. Ayarzagüena, G. Mora y J. Salas (Eds.), 150 años de Historia de la Arqueología: Teoría y Método de una disciplina (pp. 31-58). Madrid: Sociedad Española de Historia de la Arqueología.

"Salerno, P. (2012). Pensar la arqueología desde el sur. Complutum, 23(2), 191-203.

"Salmon, M. (1982). Philosophy and Archaeology. Nueva York: Academic Press.

"Schaedel, R. y Shimada, I. (1982). Peruvian Archaeology, 1946-80: An Analytic Overview. World Archaeology, 13(3), 359-371

»Schávelzon, D. (1989). The History of Mesoamerican Archaeology at the Crossroads: Changing Views of the Past". En A. Christenson (Ed.), Tracing Archaeology's past: The Historiography of Archaeology (pp. 107-112). Carbondale: Southern Illinois University Press

"Schávelzon, D. (2006). Gordon Willey y su Historia de la arqueología americana a treinta años de distancia: una mirada a América Latina, lo que había y lo que hay. En D. Schávelzon y E. King (Co-organizadores), The Gordon R. Willey Simposium in the History of Archaeology. San Juan de Puerto Rico: 71st Annual Meeting of SAA. http://www. danielschavelzon.com.ar/? $p=522$ (Acceso: 10 de marzo, 2019)

" Schlanger, N. (2002). Ancestral Archives: explorations in the history of archaeology. Antiquity, 76(291), 127-131.

"Schlanger, N. (2004). The Past is in the Present: On the History and Archives of Archaeology. Modernism, 11(1), 165-167.

» Schlanger, N. y Nordblach, J. (Eds). (2008a). Archives, Ancestors, Practices. Archaeology in the Light of its History. Nueva York: Bergham Books.

"Schlanger, N. y Nordblach, J. (2008b). Archaeology in the Light of its Histories. En N. Schlanger y J. Nordblach (Eds.) (2008), Archives, Ancestors, Practices. Archaeology in the Light of its History (pp. 1-5). Nueva York: Bergham Books. 
» Shapin, S. (1998). Placing the View from Nowhere: Historical and Sociological Problems in the Location of Science. Transactions of the Institute of British Geographers, 23, 5-12.

»Shepherd, N., Gnecco, C. y Haber, A. (2016). Arqueologías y decolonialidad. Buenos Aires: Ediciones del Signo.

» Silverman, H. y Isbell, W. (Eds.). (2008). The Handbook of South American Archaeology. Nueva York: Springer.

» Smith, C. (Ed.). (2014). Encyclopedia of Global Archaeology. Nueva York: Springer.

»Soja, E. (1989). Postmodern Geographies. The reassertion of space in critical social theory. Londres: Verso.

» Soja, E. (2010). Cities and states in geohistory. Theory and Society, 39, 361-376

» Tantalean, H. y Aguilar, M. (Comps.). (2012). La arqueología social latinoamericana. De la teoría a la praxis. Bogotá: Universidad de los Andes.

» Trigger, B. (1984). Alternative archaeologies: nationalist, colonialist, imperialist. Man, 19, 355-370.

» Trigger, B. (1992). Historia del pensamiento arqueológico. Barcelona: Crítica.

» Trigger, B. (1994). The coming of age of the history of archaeology. Journal of Archaeological Research, 2(1), 113-136.

» Trigger, B. (2001). Historiography. En T. Murray (Ed.), Encyclopedia of Archaeology History and Discoveries (pp. 630-639). Santa Barbara: ABCCLIO, Inc.

» Trigger, B. y Glover, I. (1981). Editorial. World Archaeology, 13(2), 133-137.

» Tucker, A. (Ed.). (2008). A Companion to the Philosophy of History and Historiography. Oxford y Boston: Wiley-Blackwell.

» Ucko, P. (Ed.). (1995). Theory in archaeology. A World Perspective. Londres y Nueva York: Rouletdge.

» Vargas, I. y Sanoja, M. (1999). Archaeology as a social science. Its expression in Latin America. En G. Politis y B. Alberti (Eds.), Archaeology in Latin America (pp. 59-75). Londres y Nueva York: Rouletdge.

»Watkins, J. (2005). Through Wary Eyes: Indigenous Perspectives on Archaeology. Annual Review of Anthropology, 34, 429-449.

»Willey, G. y Sabloff, J. (1974). A History of American Archaeology. Nueva York: Thames and Hudson.

» World Archaeology (1981). Regional Traditions of Archaeological Research. World Archaeology, 13(2).

»World Archaeology (1982). Regional Traditions of Archaeological Research. World Archaeology, 13(3).

»Wylie, A. (2002). Thinking from things. Essays in the philosophy of archaeology. Berkeley: University of California Press.

»Zarankin, A. y Acuto, F. (Eds.). (1999). Sed non satiata. Teoría social en la arqueología latinoamericana contemporánea. Buenos Aires: Ediciones del Tridente.

»Zarankin, A. y Acuto, F. (2008). Sed non satiata II: acercamientos sociales en la arqueología latinoamericana. Córdoba: Encuentro Grupo Editor. 